\title{
Norms of inclusions between some spaces of analytic functions
}

\section{Adrián Llinares ${ }^{1}$}

Received: 2 September 2021 / Accepted: 10 November 2021 / Published online: 6 December 2021

(c) The Author(s) 2021

\section{Abstract}

The inclusions between the Besov spaces $B^{q}$, the Bloch space $\mathcal{B}$ and the standard weighted Bergman spaces $A_{\alpha}^{p}$ are completely understood, but the norms of the corresponding inclusion operators are in general unknown. In this work, we compute or estimate asymptotically the norms of these inclusions.

Keywords Extremal problems $\cdot$ Norm of inclusions $\cdot$ Analytic Besov spaces $\cdot$ Bloch space $\cdot$ Weighted Bergman spaces

Mathematics Subject Classification $30 \mathrm{H} 20 \cdot 30 \mathrm{H} 25 \cdot 30 \mathrm{H} 30$

\section{Introduction}

As usual, let $\mathbb{D}$ denote the open unit disk on the complex plane $\mathbb{C}$. Let $\mathcal{H}(\mathbb{D})$ be the set of all analytic functions on $\mathbb{D}$. Given $0<p<\infty, r \in[0,1)$ and $f \in \mathcal{H}(\mathbb{D})$, let $M_{p}(r, f)$ be the integral $p$-mean of $|f|$ over the circle of radius $r$,

$$
M_{p}(r, f):=\left(\frac{1}{2 \pi} \int_{0}^{2 \pi}\left|f\left(r e^{i t}\right)\right|^{p} \mathrm{~d} t\right)^{\frac{1}{p}} .
$$

Consider $d A(z)=\frac{1}{\pi} \mathrm{d} x \mathrm{~d} y=\frac{r}{\pi} \mathrm{d} r \mathrm{~d} t$, the normalized area measure in $\mathbb{D}$. For any $\alpha>-1$, we define the standard weighted Bergman space $A_{\alpha}^{p}$ as the set of all holomorphic functions $f$ such that

Communicated by Jose Bonet.

Adrián Llinares

adrian.1linares@uam.es

1 Departamento de Matemáticas, Universidad Autónoma de Madrid, 28049 Madrid, Spain 


$$
\|f\|_{A_{\alpha}^{p}}:=\left(\int_{\mathbb{D}}|f|^{p} \mathrm{~d} \mu_{\alpha}\right)^{\frac{1}{p}}=\left((\alpha+1) \int_{0}^{1} 2 r\left(1-r^{2}\right)^{\alpha} M_{p}^{p}(r, f) \mathrm{d} r\right)^{\frac{1}{p}}<\infty,
$$

where for the sake of simplicity $\mathrm{d} \mu_{\alpha}(z):=(\alpha+1)\left(1-|z|^{2}\right)^{\alpha} \mathrm{d} A(z)$. If $\alpha=0$, we will simply use the notation $A^{p}$ instead of $A_{0}^{p}$.

If $q>1$ we say that $f \in \mathcal{H}(\mathbb{D})$ belongs to the (analytic) Besov space $B^{q}$ whenever its derivative $f^{\prime}$ belongs to $A_{q-2}^{q} . B^{q}$ is a Banach space when equipped with any of the following two norms:

$$
\begin{aligned}
& \|f\|_{B^{q}, 1}:=|f(0)|+\left\|f^{\prime}\right\|_{A_{q-2}^{q}}, \\
& \|f\|_{B^{q}, 2}:=\left(|f(0)|^{q}+\left\|f^{\prime}\right\|_{A_{q-2}^{q}}^{q}\right)^{\frac{1}{q}} .
\end{aligned}
$$

Clearly the two norms are equivalent, and the following sharp inequalities hold

$$
\|f\|_{B^{q}, 2} \leq\|f\|_{B^{q}, 1} \leq 2^{\frac{q-1}{q}}\|f\|_{B^{q}, 2}, \quad \forall f \in B^{q} .
$$

If $q=1$, this definition is not appropriate since the space would only contain the constant functions, so a different definition is needed. Thus, the space $B^{1}$ is usually defined as the set of all functions $f$ such that

$$
f(z)=\sum_{k=1}^{\infty} b_{k} \varphi_{a_{k}}(z)
$$

for some $\left\{a_{k}\right\}_{k \geq 1} \subset \mathbb{D}$ and $\left\{b_{k}\right\}_{k \geq 1} \in \ell^{1}$. Here $\varphi_{a}$ is used to denote the disk automorphism

$$
\varphi_{a}(z):=\frac{a-z}{1-\bar{a} z}
$$

If $\|f\|_{B^{1}}:=\inf \left\{\left\|\left\{b_{k}\right\}_{k \geq 1}\right\|_{\ell^{1}}\right\}$, it can be shown [2] that there exist two absolute constants $A, B>0$ such that

$$
A\left\|f^{\prime \prime}\right\|_{A^{1}} \leq\left\|f-f(0)-f^{\prime}(0) z\right\|_{B^{1}} \leq B\left\|f^{\prime \prime}\right\|_{A^{1}}, \quad \forall f \in B^{1},
$$

and consequently an alternative characterization of $B^{1}$ can be given in terms of the integrability of the second derivative, similarly to the way of defining $B^{q}$ for $q>1$. Here and also elsewhere in the paper, we will also write (2) as

$$
\left\|f-f(0)-f^{\prime}(0) z\right\|_{B^{1}} \approx\left\|f^{\prime \prime}\right\|_{A^{1}} .
$$

If only one inequality holds, we will use the symbol $\lesssim$ or $\gtrsim$ instead.

Finally, the Bloch space $\mathcal{B}$ is the class of all the analytic functions such that

$$
\rho_{\mathcal{B}}(f):=\sup _{z \in \mathbb{D}}\left\{\left|f^{\prime}(z)\right|\left(1-|z|^{2}\right)\right\}<\infty .
$$


The expression $\rho_{\mathcal{B}}$ is actually a (complete) seminorm, and thus $\mathcal{B}$ is a Banach space with respect to the norm

$$
\|f\|_{\mathcal{B}}:=|f(0)|+\rho_{\mathcal{B}}(f)
$$

The Bloch space is not separable, and therefore, the closure of the polynomials is a proper subspace of $\mathcal{B}$. This subspace is called the little Bloch space $\mathcal{B}_{0}$. It can be proven [1] that

$$
f \in \mathcal{B}_{0} \Longleftrightarrow \lim _{|z| \rightarrow 1^{-}}\left\{\left|f^{\prime}(z)\right|\left(1-|z|^{2}\right)\right\}=0
$$

As a last word regarding the notation, we are going to write

$$
B(x, y):=\int_{0}^{1} t^{x-1}(1-t)^{y-1} \mathrm{~d} t \quad \text { and } \quad \Gamma(x):=\int_{0}^{\infty} t^{x-1} e^{-t} \mathrm{~d} t, \quad x, y>0
$$

to denote the Euler Beta and Gamma functions, respectively.

It is well known that the following chain of inclusions holds

$$
B^{q} \subset \mathcal{B} \subset A_{\alpha}^{p}, \quad \text { if } q \geq 1, p>0 \text { and } \alpha>-1,
$$

hence, as a consequence of closed graph theorem $\left(A_{\alpha}^{p}\right.$ is not a normed space if $p<1$, but it is complete and invariant under translations also in this case) the inclusion operators between them are bounded. The norm of these inclusions are in general either not known or difficult to find in the literature.

Since the inclusions between weighted Bergman spaces are well understood, we can check that $B^{q} \subset B^{p}$ if $p>q$. The norm of this inclusion operator is conjectured to be 1 (see for example [3]), but the tools needed to study this problem are completely different than the used in this work. Therefore, this inclusion will not be covered in this paper.

We are interested in estimating as precisely as possible the norms of the two inclusions in (3) (the third inclusion $B^{q} \subset A_{\alpha}^{p}$ can be trivially estimated by composition).

More specifically, the main results of this work can be listed as follows:

- $\|f\|_{\mathcal{B}} \leq\|f\|_{B^{q}, 1}$ and $\|f\|_{\mathcal{B}} \leq 2^{\frac{q-1}{q}}\|f\|_{B^{q}, 2}$.

- $\|f\|_{\mathcal{B}} \leq 2\|f\|_{B^{1}}$.

- If $p \geq 1, \sup \left\{\|f\|_{A_{\alpha}^{p}}:\|f\|_{\mathcal{B}} \leq 1\right\} \approx p$.

- If $\alpha \geq 0$, there exists $p_{\alpha}>2 \max \left\{1, B^{-1}\left(\frac{1}{2}, \alpha+1\right)\right\}$ such that $\|f\|_{A_{\alpha}^{p}} \leq\|f\|_{\mathcal{B}}$ for every $p \in\left(0, p_{\alpha}\right]$.

\section{The inclusion of $B^{q}$ in $\mathcal{B}$}

There are many ways of proving that $B^{q} \subset \mathcal{B}$. Maybe the most elegant one is to show that $B^{q}$ is conformally invariant and using the fact that $\mathcal{B}$ is (under some conditions) maximal in this class of spaces [8], as a counterpart of $B^{1}$, which is minimal [2]. 
However, it is easier to prove that $B^{q} \subset \mathcal{B}$ via the pointwise bounds of $A_{\alpha}^{p}$ functions (see [9] or Lemma 3.2 in [6], for example).

Lemma 1 (Pointwise estimates in $A_{\alpha}^{p}$ ). If $p>0, \alpha>-1$ and $\zeta \in \mathbb{D}$, then

$$
|g(\zeta)| \leq \frac{\|g\|_{A_{\alpha}^{p}}}{\left(1-|\zeta|^{2}\right)^{\frac{\alpha+2}{p}}},
$$

for all $g \in A_{\alpha}^{p}$.

Moreover, equality holds if and only if $g$ is a multiple of

$$
k_{\zeta}(z):=\frac{1}{\left(1-\bar{\zeta}_{z}\right)^{\frac{2(\alpha+2)}{p}}}, \quad z \in \mathbb{D} .
$$

If $f \in B^{q}$ for some $q>1$, then

$$
\left|f^{\prime}(z)\right|\left(1-|z|^{2}\right) \leq\left\|f^{\prime}\right\|_{A_{q-2}^{q}}, \quad \forall z \in \mathbb{D},
$$

and, therefore, $f \in \mathcal{B}$.

On the other hand, if $f \in B^{1}$ then $f$ is indeed a bounded analytic function and due to Schwarz-Pick lemma (after a suitable normalization), we get that $f \in \mathcal{B}$.

At this point, we are able to compute the norm of the inclusion $B^{q} \subset \mathcal{B}$.

\section{Proposition 2.1}

1. Let $q>1$. Then,

$$
\|f\|_{\mathcal{B}} \leq\|f\|_{B^{q}, 1} \quad \text { and } \quad\|f\|_{\mathcal{B}} \leq 2^{\frac{q-1}{q}}\|f\|_{B^{q}, 2},
$$

for all $f \in B^{q}$.

Moreover, if the equality is attained in any of the above inequalities, then $f$ must be of one of the following forms

$$
\begin{aligned}
& f_{0}(z):=\alpha z+\beta \\
& f_{\zeta}(z):=\gamma \frac{1-|\zeta|^{2}}{\bar{\zeta}} \frac{1}{1-\bar{\zeta} z}+\delta, \quad 0<|\zeta|<1,
\end{aligned}
$$

for some suitable constants $\alpha, \beta, \gamma$ and $\delta$.

2. The following inequality is sharp

$$
\|f\|_{\mathcal{B}} \leq 2\|f\|_{B^{1}} .
$$

Moreover, equality is attained only if $f \equiv 0$. 


\section{Proof}

1. If $f \in B^{q}$, clearly

$\|f\|_{\mathcal{B}}=|f(0)|+\rho_{\mathcal{B}}(f) \leq|f(0)|+\left\|f^{\prime}\right\|_{A_{q-2}^{q}}=\|f\|_{B^{q}, 1} \leq 2^{\frac{q-1}{q}}\|f\|_{B^{q}, 2}$.

Observe that if either $\|f\|_{\mathcal{B}}=\|f\|_{B^{p}, 1}$ or $\|f\|_{\mathcal{B}}=2^{\frac{q-1}{q}}\|f\|_{B^{q}, 2}$, then necessarily $\rho_{\mathcal{B}}(f)=\left\|f^{\prime}\right\|_{A_{q-2}^{q}}$. Note that the polynomials are dense in $B^{q}$ and the inclusion operator is continuous, so indeed $B^{q} \subset \mathcal{B}_{0}$. Thus, there exists $\zeta \in \mathbb{D}$ such that

$$
\left|f^{\prime}(\zeta)\right|\left(1-|\zeta|^{2}\right)=\rho_{\mathcal{B}}(f)=\left\|f^{\prime}\right\|_{A_{q-2}^{q}},
$$

and, therefore, we have that $f^{\prime}(z)=c k_{\zeta}(z)=c\left(1-\bar{\zeta}_{z}\right)^{-2}$ for some constant $c$, from where we deduce the expression of $f$.

2. Let $f \in B^{1}$ such that $\|f\|_{B^{1}}=1$. As is usual, let $\|f\|_{\infty}:=\sup \{|f(z)|: z \in \mathbb{D}\}$. If $\left\{b_{k}\right\}_{k \geq 1}$ is an admissible sequence of coefficients for $f$ in the formula (1), then it is clear that $\|f\|_{\infty} \leq\left\|\left\{b_{k}\right\}_{k \geq 1}\right\|_{\ell^{1}}$, so $\|f\|_{\infty} \leq\|f\|_{B^{1}}$. Thus, we can apply the Schwarz-Pick lemma to the function $\frac{f}{\|f\|_{\infty}}$ in order to get $\left|f^{\prime}(z)\right|\left(1-|z|^{2}\right) \leq\|f\|_{\infty}$ for every $z$ in $\mathbb{D}$.

Summing up,

$$
\|f\|_{\mathcal{B}}=|f(0)|+\rho_{\mathcal{B}}(f) \leq 2\|f\|_{\infty} \leq 2 .
$$

This inequality is sharp, because if we test with the sequence of automorphisms $\left\{\varphi_{1-k^{-1}}\right\}_{k \geq 1}$, it is immediate to check that $\left\|\varphi_{1-k^{-1}}\right\|_{B^{1}}=1$ and

$$
\left\|\varphi_{1-\frac{1}{k}}\right\|_{\mathcal{B}}=2-\frac{1}{k}, \quad k \geq 1 .
$$

However, if equality holds for a non-identically zero function $f$ (whose $B^{1}$ norm is assumed to be 1 ), then in particular $\|f\|_{\mathcal{B}}=2\|f\|_{\infty}=2$. This leads to

$$
|f(0)|=\rho_{\mathcal{B}}(f)=\|f\|_{\infty}=1,
$$

which is in a clear contradiction with the open mapping theorem. Therefore, there are no extremal functions for this inclusion.

\section{Remark 2.2}

1. It can be checked that $\left\|f_{\zeta}\right\|_{\mathcal{B}}=\left\|f_{\zeta}\right\|_{B^{q}, 1}, \zeta \in \mathbb{D}$, for any choice of $\alpha, \beta, \gamma$ and $\delta$.

On the other hand, $\left\|f_{\zeta}\right\|_{\mathcal{B}}=2^{\frac{q-1}{q}}\left\|f_{\zeta}\right\|_{B^{q}, 2}$, whenever $|\alpha|=|\beta|=2^{-\frac{1}{q}}\left\|f_{0}\right\|_{B^{q}, 2}$, if $\zeta=0$, or $|\gamma|=\left|\gamma \frac{1-|\zeta|^{2}}{\bar{\zeta}}+\delta\right|=2^{-\frac{1}{q}}\left\|f_{\zeta}\right\|_{B^{q}, 2}$ otherwise.

2. The inequality $\|f\|_{\mathcal{B}} \leq 2\|f\|_{\infty}$ is widely known and can be found in many references (like the remarkable work of Anderson, Clunie and Pommerenke [1]), but 
the non-existence of extremal functions, although rather straightforward, is not explicitly stated in the literature.

\section{The inclusion of $\mathcal{B}$ in $A_{\alpha}^{p}$}

As a consequence of the definition of the seminorm, we have that

$$
|f(z)-f(0)| \leq \frac{1}{2} \log \left(\frac{1+|z|}{1-|z|}\right) \rho_{\mathcal{B}}(f), \quad \forall z \in \mathbb{D} .
$$

This growth at most logarithmic yields that $\mathcal{B} \subset A_{\alpha}^{p}$ for every $p>0$ and $\alpha>-1$. Moreover, it is sufficient to prove the compactness of the inclusion operator.

Lemma 3.1 The inclusion of $\mathcal{B}$ in $A_{\alpha}^{p}$ is a compact operator.

Proof Let $\left\{f_{n}\right\}_{n \geq 1}$ a sequence in $\mathcal{B}$ such that $\left\|f_{n}\right\|_{\mathcal{B}} \leq 1$. As a consequence of (4), $\left\{f_{n}\right\}_{n \geq 1}$ is uniformly bounded on compact sets of $\mathbb{D}$, and therefore there exists a subsequence $\left\{f_{n_{k}}\right\}_{k \geq 1}$ uniformly convergent on compact sets to some analytic function $f$.

Note that

$$
\left|f_{n_{k}}(z)\right|^{p} \leq\left[1+\frac{1}{2} \log \left(\frac{1+|z|}{1-|z|}\right)\right]^{p}, \quad \forall z \in \mathbb{D},
$$

and the right-hand side of this last inequality is integrable with respect the measure $\mu_{\alpha}$, hence due to the dominated convergence theorem it follows that $\left\|f_{n_{k}}\right\|_{A_{\alpha}^{p}}$ converges to $\|f\|_{A_{\alpha}^{p}}$. This convergence of the $A_{\alpha}^{p}$ norms and the convergence $\mu_{\alpha}$-a.e. are enough, using the Riesz's lemma (see [4], Lemma 1 in Chapter 2), to prove that $\lim _{k \rightarrow \infty} f_{n_{k}}=f$ in the $A_{\alpha}^{p}$ topology.

For the sake of simplicity, denote

$$
C_{\alpha}(p):=\max \left\{\|f\|_{A_{\alpha}^{p}}:\|f\|_{\mathcal{B}} \leq 1\right\} .
$$

Note that the maximum in (5) is attained, because the inclusion is a compact operator.

Given $f \in \mathcal{H}(\mathbb{D})$, due to an elementary fact from Measure Theory

$$
\lim _{p \rightarrow \infty}\|f\|_{A_{\alpha}^{p}}=\|f\|_{\infty},
$$

so the value $C_{\alpha}(p)$ must blow up whenever $p$ tends to infinity.

First, we are going to deduce the order of growth of $C_{\alpha}(p)$. The following lemma will be needed.

Lemma 2 (Chebyshev's inequality) Let $\sigma$ a probability measure on a real interval $(a, b)$. If $f$ and $g$ are two increasing functions such that $f, g$ and fg are integrable with respect $\sigma$, then 


$$
\int_{a}^{b} f g \mathrm{~d} \sigma \geq \int_{a}^{b} f \mathrm{~d} \sigma \int_{a}^{b} g \mathrm{~d} \sigma
$$

Proof The proof is a direct consequence of the fact that the function $(f(x)-f(y))(g(x)-g(y))$ is non-negative and Fubini's theorem.

Theorem 3.2 Let $\alpha>-1$ fixed. If $p \geq 1$, then $C_{\alpha}(p) \approx p$.

Proof Take $f \in \mathcal{B}$, and assume that $f(0)=0$.

Consider the probability measure $\mathrm{d} \sigma(r):=\frac{2\left(1-r^{2}\right)^{\alpha} \mathrm{d} r}{B\left(\frac{1}{2}, \alpha+1\right)}$. Note that

$$
\int_{0}^{1} 2 r \mathrm{~d} \sigma(r)=\frac{2}{(\alpha+1) B\left(\frac{1}{2}, \alpha+1\right)} .
$$

Due to the inequality (see for example [4], page 82)

$$
\frac{\mathrm{d}}{\mathrm{d} r} M_{p}^{p}(r, f) \leq p M_{p}^{p-1}(r, f) M_{p}\left(r, f^{\prime}\right), \quad \forall r \in(0,1),
$$

integrating by parts and using Chebyshev's inequality, it follows that

$$
\begin{aligned}
\|f\|_{A_{\alpha}^{p}}^{p} & =\int_{0}^{1}\left(1-r^{2}\right)^{\alpha+1} \frac{\mathrm{d}}{\mathrm{d} r} M_{p}^{p}(r, f) \mathrm{d} r \\
& \leq \mathrm{d} \frac{B\left(\frac{1}{2}, \alpha+1\right)}{2} p\left(\int_{0}^{1} M_{p}^{p-1}(r, f) \mathrm{d} \sigma(r)\right)\|f\|_{\mathcal{B}} \\
& =\left(\frac{B\left(\frac{1}{2}, \alpha+1\right)}{2}\right)^{2} p(\alpha+1)\left(\int_{0}^{1} 2 r \mathrm{~d} \sigma(r)\right)\left(\int_{0}^{1} M_{p}^{p-1}(r, f) \mathrm{d} \sigma(r)\right)\|f\|_{\mathcal{B}} \\
& \leq \frac{B\left(\frac{1}{2}, \alpha+1\right)}{2} p(\alpha+1)\left(\int_{0}^{1} 2 r\left(1-r^{2}\right)^{\alpha} M_{p}^{p-1}(r, f) \mathrm{d} r\right)\|f\|_{\mathcal{B}},
\end{aligned}
$$

and the estimate $\|f\|_{A_{\alpha}^{p}} \leq \frac{B\left(\frac{1}{2}, \alpha+1\right)}{2} p\|f\|_{\mathcal{B}}$, if $f(0)=0$, follows from Hölder's inequality.

The Bloch seminorm $\rho_{B}$ is invariant under translations. Thus, for every $f \in \mathcal{B}$

$$
\|f\|_{A_{\alpha}^{p}} \leq|f(0)|+\|f-f(0)\|_{A_{\alpha}^{p}} \leq \max \left\{\frac{B\left(\frac{1}{2}, \alpha+1\right)}{2}, 1\right\} p\|f\|_{\mathcal{B}} .
$$

That is, $C_{\alpha}(p) \lesssim p$. 
To get the reverse inequality, we are going to test with the function $f(z)=-\frac{1}{2} \log (1-z)$. It is immediate to check that $\|f\|_{\mathcal{B}}=1$ and using polar coordinates centered at 1

$$
\begin{aligned}
\|f\|_{A_{\alpha}^{p}}^{p} & \geq \frac{\alpha+1}{\pi 2^{p}} \int_{\frac{\pi}{2}}^{\frac{3 \pi}{2}} \int_{0}^{2|\cos t|} r^{\alpha+1}(2|\cos t|-r)^{\alpha}\left|\log \frac{1}{r}\right|^{p} \mathrm{~d} r \mathrm{~d} t \\
& \geq \frac{\alpha+1}{\pi 2^{p-1}} \int_{\frac{2 \pi}{3}}^{\pi} \int_{0}^{1} r^{\alpha+1}(2|\cos t|-r)^{\alpha} \log ^{p} \frac{1}{r} \mathrm{~d} r \mathrm{~d} t .
\end{aligned}
$$

Observe that the factor $(2|\cos t|-r)^{\alpha}$ is a monotonic function with respect $r$, so if we write

$$
M_{\alpha}:=\frac{1}{\pi} \int_{\frac{2 \pi}{3}}^{\pi} \min \left\{(2|\cos t|)^{\alpha},(2|\cos t|-1)^{\alpha}\right\} \mathrm{d} t
$$

then

$$
C_{\alpha}^{p}(p) \geq\|f\|_{A_{\alpha}^{p}}^{p} \geq \frac{M_{\alpha}}{2^{p-1}} \int_{0}^{1} r^{\alpha+1} \log ^{p} \frac{1}{r} \mathrm{~d} r=\frac{M_{\alpha}}{2^{p-1}(\alpha+2)^{p+1}} \Gamma(p+1) .
$$

Consequently, the inequality $C_{\alpha}(p) \gtrsim p$ holds due to Stirling's formula.

Indeed, the proof above can be slightly modified to demonstrate the contractivity of the inclusion for a wide range of exponents (depending on $\alpha$, of course).

Theorem 3.3 Let $\alpha \geq 0$. If $p \leq \frac{2}{B\left(\frac{1}{2}, \alpha+1\right)}$, then

$$
\|f\|_{A_{\alpha}^{p}} \leq\|f\|_{\mathcal{B}}
$$

for every $f \in \mathcal{B}$.

In addition, the equality is attained if and only iff is constant.

In particular, $C_{\alpha}(p)=1$ for all these values of $p$.

Proof Note that $\mu_{\alpha}$ is a probability measure, so it is enough to prove the contractivity for $p=\frac{2}{B\left(\frac{1}{2}, \alpha+1\right)}$. This quantity is strictly increasing in $\alpha$, and therefore $p \geq 1$ because of $\alpha \geq 0$. Then,

$$
\|f\|_{A_{\alpha}^{p}} \leq|f(0)|+\|f-f(0)\|_{A_{\alpha}^{p}} \leq|f(0)|+\frac{B\left(\frac{1}{2}, \alpha+1\right)}{2} p \rho_{\mathcal{B}}(f)=\|f\|_{\mathcal{B}} .
$$

If equality holds then the Bloch seminorm is attained at every point. That is, 


$$
\left|f^{\prime}(z)\right|=\frac{\rho_{\mathcal{B}}(f)}{1-|z|^{2}}, \quad \forall z \in \mathbb{D}
$$

but an elementary application of the maximum modulus principle and the identity principle shows that the only analytic functions whose moduli are radial functions are the monomials, hence necessarily $\rho_{\mathcal{B}}(f)=0$ and $f$ is constant.

However, this range of contractivity is not, in general, sharp. We are going to prove that, por any $\alpha \geq 0$ the inclusion of $\mathcal{B}$ in the Hilbert space $A_{\alpha}^{2}$ is also contractive (a fact that it is not covered in the last theorem for $\alpha$ small enough).

A sketch of the proof for the unweighted case $\alpha=0$ can be found in [1], where the expression of $\|f\|_{A^{2}}$ in terms of the Taylor coefficients of $f$ is needed. In the general case, our main idea relies upon the so called Hardy-Stein identity ([7], Section 8.2), or simply Hardy identity in some references,

$$
\frac{\mathrm{d}}{\mathrm{d} r} M_{p}^{p}(r, f)=\frac{p^{2}}{2 r} \int_{r \mathbb{D}}\left|f^{\prime}(z)\right|^{2}|f(z)|^{p-2} d A(z), \quad \forall r \in(0,1),
$$

which can be understood as a refinement of (6).

Theorem 3.4 If $\alpha \geq 0$, then

$$
\|f\|_{A_{\alpha}^{2}} \leq\|f\|_{\mathcal{B}}
$$

and equality is attained only for constant functions.

Proof Take $f \in \mathcal{B}$. Using integration by parts and the Hardy-Stein identity, we obtain

$$
\begin{aligned}
\|f\|_{A_{\alpha}^{2}}^{2} & =|f(0)|^{2}+2 \int_{0}^{1} \frac{\left(1-r^{2}\right)^{\alpha+1}}{r} \int_{r \mathbb{D}}\left|f^{\prime}(z)\right|^{2} \mathrm{~d} A(z) \mathrm{d} r \\
& \leq|f(0)|^{2}+\frac{\rho_{\mathcal{B}}(f)^{2}}{\alpha+1} \leq\|f\|_{\mathcal{B}}^{2} .
\end{aligned}
$$

Again, $\|f\|_{A_{\alpha}^{2}}=\|f\|_{\mathcal{B}}$ implies that $\rho_{\mathcal{B}}(f)$ is attained at every point, and thus $f$ is constant.

At this point, we realize that the functions that vanish at the origin play an important role, and consequently we are going to write $\tilde{C}_{\alpha}(p)$ to denote the extremal problem

$$
\tilde{C}_{\alpha}(p)=\max \left\{\|f\|_{A_{\alpha}^{p}}:\|f\|_{\mathcal{B}} \leq 1, f(0)=0\right\} .
$$

As an initial remark, note that Theorem 3.2 holds also for $\tilde{C}_{\alpha}(p)$. That is, $\tilde{C}_{\alpha}(p) \approx p$ as well.

It is trivial that $\tilde{C}_{\alpha}(p) \leq C_{\alpha}(p)$ for every $p$, but if both $p$ and $\tilde{C}_{\alpha}(p)$ are greater than or equal to 1 , due to the triangle inequality 


$$
\|f\|_{A_{\alpha}^{p}} \leq|f(0)|+\tilde{C}_{\alpha}(p) \rho_{\mathcal{B}}(f) \leq \tilde{C}_{\alpha}(p)\|f\|_{\mathcal{B}},
$$

and therefore $C_{\alpha}(p)=\tilde{C}_{\alpha}(p)$ in this case.

Similarly, $\tilde{C}_{\alpha}(p)<1$ implies that $C_{\alpha}(p)=1$. In other words,

$$
C_{\alpha}(p)=\max \left\{1, \tilde{C}_{\alpha}(p)\right\},
$$

for any $p \geq 1$.

Observe that $\tilde{C}_{\alpha}$ is strictly increasing due to the existence of extremal functions and Hölder's inequality.

Corollary 3.5 If $\alpha \geq 0$, there exists $p_{\alpha}>2 \max \left\{1, B^{-1}\left(\frac{1}{2}, \alpha+1\right)\right\}$ such that

$$
\|f\|_{A_{\alpha}^{p}} \leq\|f\|_{\mathcal{B}},
$$

for any $p \in\left(0, p_{\alpha}\right]$.

Proof It is a consequence of (7) and Theorems 3.3 and 3.4.

However, this corollary does not imply that this improvement is "significantly" large. In the unweighted case, the model function $f(z)=\frac{1}{2} \log \frac{1+z}{1-z}$ verifies, after some suitable changes of variables, that

$$
\|f\|_{A^{\frac{25}{4}}}^{\frac{25}{4}}=\frac{1}{\pi 2^{\frac{9}{4}}} \int_{0}^{\infty} \int_{0}^{\frac{\pi}{2}} \frac{\left(x^{2}+y^{2}\right)^{\frac{25}{8}}}{\left(e^{x}+2 \cos (y)+e^{-x}\right)^{2}} \mathrm{~d} y \mathrm{~d} x>1,
$$

and therefore $p_{0} \in\left(2, \frac{25}{4}\right)$. This last inequality has been checked by a numerical computation using the software Wolfram Alpha.

Next, since $C_{\alpha}$ and $\tilde{C}_{\alpha}$ will be eventually equal, we are going to exploit the restriction of $\tilde{C}_{\alpha}$ in order to guess the correct asymptotics for $C_{\alpha}$.

Lemma 3.6 If $\alpha>-1$ and $f(z)=\sum_{n=0}^{\infty} a_{n} z^{n}$ is analytic in $\mathbb{D}$, then

$$
(\alpha+1)(\alpha+2) \sum_{n=1}^{\infty} \frac{n}{n+\alpha+2} \frac{\Gamma(\alpha+2) n !}{\Gamma(n+\alpha+2)}\left|a_{n}\right|^{2}=\int_{\mathbb{D}}\left|f^{\prime}(z)\right|^{2}\left(1-|z|^{2}\right)^{2} \mathrm{~d} \mu_{\alpha}(z) .
$$

In addition, if $f(0)=\cdots=f^{(k-1)}(0)=0$ for some $k \geq 1$,

$$
\|f\|_{A_{\alpha}^{2}}^{2} \leq \frac{k+\alpha+2}{(\alpha+1)(\alpha+2) k} \int_{\mathbb{D}}\left|f^{\prime}(z)\right|^{2}\left(1-|z|^{2}\right)^{2} \mathrm{~d} \mu_{\alpha}(z) .
$$

Proof This is a consequence of Parseval's identity and the monotonicity of the sequence $\left\{\frac{n}{n+\alpha+2}\right\}_{n \geq 1}$.

Theorem 3.7 For any $\alpha>-1$ and $n \geq 2$ 


$$
\tilde{C}_{\alpha}(2 n) \leq \frac{1}{\sqrt{(\alpha+1)(\alpha+2)}}\left(\frac{(\alpha+1)(\alpha+2) \Gamma(n+\alpha+3) n !}{\Gamma(\alpha+4)} \tilde{C}_{\alpha}^{2}(2)\right)^{\frac{1}{2 n}} .
$$

Proof We give a proof by Induction. Let $f \in \mathcal{B}$ with $f(0)=0$ and $\|f\|_{\mathcal{B}}=1$. Note that $f^{2}$ verifies that $f^{2}(0)=\left(f^{2}\right)^{\prime}(0)=0$ and due to Lemma 3.6

$$
\begin{aligned}
\|f\|_{A_{\alpha}^{4}}^{4}=\left\|f^{2}\right\|_{A_{\alpha}^{2}}^{2} & \leq \frac{\alpha+4}{2(\alpha+1)(\alpha+2)} \int_{\mathbb{D}}\left|\left(f^{2}\right)^{\prime}(z)\right|^{2}\left(1-|z|^{2}\right)^{2} \mathrm{~d} \mu_{\alpha}(z) \\
& \leq \frac{2(\alpha+4)}{(\alpha+1)(\alpha+2)}\|f\|_{A_{\alpha}^{2}}^{2} \leq \frac{2(\alpha+4)}{(\alpha+1)(\alpha+2)} \tilde{C}_{\alpha}^{2}(2),
\end{aligned}
$$

and hence the statement has been proved for $n=2$.

Now assume

$$
\tilde{C}_{\alpha}(2 n) \leq \frac{1}{\sqrt{(\alpha+1)(\alpha+2)}}\left(\frac{(\alpha+1)(\alpha+2) \Gamma(n+\alpha+3) n !}{\Gamma(\alpha+4)} \tilde{C}_{\alpha}^{2}(2)\right)^{\frac{1}{2 n}},
$$

for some $n \geq 2$.

Again, it is immediate that the first $n+1$ Taylor coefficients of $f^{n+1}$ are zero and therefore

$$
\begin{aligned}
\|f\|_{A_{\alpha}^{2(n+1)}}^{2(n+1)}=\left\|f^{n+1}\right\|_{A_{\alpha}^{2}}^{2} & \leq \frac{n+\alpha+3}{(\alpha+1)(\alpha+2)(n+1)} \int_{\mathbb{D}}\left|f^{(n+1)}(z)\right|^{2}\left(1-|z|^{2}\right)^{2} \mathrm{~d} \mu_{\alpha}(z) \\
& \leq \frac{(n+\alpha+3)(n+1)}{(\alpha+1)(\alpha+2)}\|f\|_{A^{2 n}}^{2 n} \\
& \leq \frac{\Gamma(n+\alpha+4)(n+1) !}{(\alpha+1)^{n}(\alpha+2)^{n} \Gamma(\alpha+4)} \tilde{C}_{\alpha}^{2}(2),
\end{aligned}
$$

which proves the theorem.

Corollary 3.8 If $\alpha>-1$, then

$$
\begin{aligned}
\liminf _{p \rightarrow \infty} \frac{C_{\alpha}(p)}{p} \geq \frac{1}{2 e(\alpha+2)}, \\
\limsup _{p \rightarrow \infty} \frac{C_{\alpha}(p)}{p} \leq \frac{1}{2 e \sqrt{(\alpha+1)(\alpha+2)}} .
\end{aligned}
$$

Proof The lower bound for the limit inferior can be deduced from the proof of Theorem 3.2 and, of course, Stirling's formula for $\Gamma$.

On the other hand, if $p$ is large enough

$$
\frac{C_{\alpha}(p)}{p}=\frac{\tilde{C}_{\alpha}(p)}{p} \leq \frac{2\left\lceil\frac{p}{2}\right\rceil}{p} \frac{\tilde{C}_{\alpha}\left(2\left\lceil\frac{p}{2}\right\rceil\right)}{2\left\lceil\frac{p}{2}\right\rceil}
$$


where $\lceil\cdot\rceil$ is the greatest integer part, so we can use Theorem 3.7 to get the upper bound for the limit superior.

Observe that both bounds are asymptotically equivalent if $\alpha$ tends to infinity, so there is the strong feeling that the quotient $\frac{C_{\alpha}(p)}{p}$ might be convergent with respect $p$, not only bounded.

Moreover, it is widely know that $\mathcal{B}$ is not contained in any Hardy space $H^{p}$ (that is, the set of all analytic functions whose $M_{p}$ means are uniformly bounded), which can be understood as the limit space $A_{-1}^{p}$ in the sense that

$$
\|f\|_{H^{p}}=\lim _{\alpha \rightarrow-1^{+}}\|f\|_{A_{\alpha}^{p}} .
$$

Thus, the accurate asymptotic approximation should blow up whenever $\alpha$ tends to -1 . Given these considerations, we conjecture that $\frac{C_{\alpha}(p)}{p}$ is actually convergent for any $\alpha>-1$ and the limit should coincide with the bound for the limit superior given in Corollary 3.8.

Conjecture 3.9 For any $\alpha>-1$,

$$
\lim _{p \rightarrow \infty} \frac{C_{\alpha}(p)}{p /(2 e \sqrt{(\alpha+1)(\alpha+2)})}=1 .
$$

We end the paper by deducing an integral condition that the extremal functions must verify.

Theorem 3.10 Let $\alpha>-1, p>1$ and $f \in \mathcal{B}$ non-identically zero such that $f(0)=0$ and $\|f\|_{A_{\alpha}^{p}}=\tilde{C}_{\alpha}(p)\|f\|_{\mathcal{B}}$. Then $f$ is a solution of the functional equation

$$
\int_{\mathbb{D}}|f(z)|^{p} z \mathrm{~d} \mu_{\alpha}(z)=\frac{p}{2(\alpha+2)} \overline{f^{\prime}(0)} \int_{\mathbb{D}}|f(z)|^{p-2} f(z) \mathrm{d} \mu_{\alpha}(z) .
$$

Proof Take $f \in \mathcal{B}, f \not \equiv 0$, such that $f(0)=0$ and $\|f\|_{A_{\alpha}^{p}}=\tilde{C}_{\alpha}(p)\|f\|_{\mathcal{B}}$.

Consider the family of functions

$$
g_{a}(z):=f\left(\varphi_{a}(z)\right)-f(a), \quad a \in \mathbb{D} .
$$

It is clear that $g_{a}(0)=0$ for every $a$, and the Bloch space is strictly conformally invariant (i.e., $\rho_{\mathcal{B}}\left(h \circ \varphi_{a}\right)=\rho_{\mathcal{B}}(h)$ whenever $\left.h \in \mathcal{B}\right)$ so $\left\|g_{a}\right\|_{\mathcal{B}}=\|f\|_{\mathcal{B}}, \forall a \in \mathbb{D}$. The function $f$ is extremal and then by definition

$$
\int_{\mathbb{D}}|f(\zeta)-f(a)|^{p}\left|\varphi_{a}^{\prime}(\zeta)\right|^{\alpha+2} \mathrm{~d} \mu_{\alpha}(\zeta)=\int_{\mathbb{D}}\left|g_{a}\right|^{p} \mathrm{~d} \mu_{\alpha} \leq \int_{\mathbb{D}}|f|^{p} \mathrm{~d} \mu_{\alpha} .
$$

Note that $\left|\varphi_{a}^{\prime}(\zeta)\right|^{2}=\frac{\left(1-|a|^{2}\right)^{2}}{\mid 1-\bar{a} \zeta}=1+4 \operatorname{Re}\{\bar{a} \zeta\}+o(|a|)$, and therefore

$$
\left|\varphi_{a}^{\prime}(\zeta)\right|^{\alpha+2}=1+2(\alpha+2) \operatorname{Re}\{\bar{a} \zeta\}+o(|a|), \quad a \rightarrow 0 .
$$

Take the partition $\mathbb{D}_{a}^{+}:=\{\zeta \in \mathbb{D}:|f(\zeta)|>|f(a)|\}$ and $\mathbb{D}_{a}^{-}:=\mathbb{D} \backslash \mathbb{D}_{a}^{+}$. 
- If $\zeta \in \mathbb{D}_{a}^{-}$, using the fact that $p>1$ and $f(0)=0$,

$$
|f(\zeta)-f(a)|^{p} \leq 2^{p}|f(a)|^{p}=o(|a|), \quad a \rightarrow 0 .
$$

Thus, applying the dominated convergence theorem we have

$$
\begin{aligned}
\int_{\mathbb{D}_{a}^{-}}|f(\zeta)-f(a)|^{p}\left|\varphi_{a}^{\prime}(\zeta)\right|^{\alpha+2} \mathrm{~d} \mu_{\alpha}(\zeta) & =o(|a|), \\
\int_{\mathbb{D}_{a}^{-}}|f|^{p} \mathrm{~d} \mu_{\alpha} & =o(|a|),
\end{aligned}
$$

for $a \rightarrow 0$.

- If $\zeta \in \mathbb{D}_{a}^{+}$,

$$
|f(\zeta)-f(a)|^{p}=|f(\zeta)|^{p}-p|f(\zeta)|^{p-2} \operatorname{Re}\left\{f(\zeta) \overline{f^{\prime}(0) a}\right\}+o(|a|), \quad a \rightarrow 0 .
$$

Applying again the dominated convergence theorem,

$$
\begin{aligned}
\int_{\mathbb{D}_{a}^{+}}|f-f(a)|^{p}\left|\varphi_{a}^{\prime}\right|^{\alpha+2} \mathrm{~d} \mu_{\alpha}= & \int_{\mathbb{D}_{a}^{+}}|f|^{p-2} \operatorname{Re}\left\{\left[2(\alpha+2)|f|^{2} \zeta-p f \overline{f^{\prime}(0)}\right] \bar{a}\right\} \mathrm{d} \mu_{\alpha} \\
& +\int_{\mathbb{D}_{a}^{+}}|f|^{p} \mathrm{~d} \mu_{\alpha}+o(|a|), \quad a \rightarrow 0 .
\end{aligned}
$$

Summing up, if we write $a=r e^{i t}$ with $r>0$ and $t \in[0,2 \pi)$, we have

$$
\operatorname{Re}\left\{e^{-i t} \int_{\mathbb{D}_{a}^{+}}|f(\zeta)|^{p-2}\left[2(\alpha+2)|f(\zeta)|^{2} \zeta-p \overline{f^{\prime}(0)} f(\zeta)\right] \mathrm{d} \mu_{\alpha}(\zeta)\right\} \leq \frac{o(r)}{r}, \quad r \rightarrow 0^{+} .
$$

Using one last time the dominated convergence theorem, we prove

$$
\int_{\mathbb{D} \backslash \mathcal{Z}(f)}|f(\zeta)|^{p} \zeta \mathrm{d} \mu_{\alpha}(\zeta)=\frac{p}{2(\alpha+2)} \overline{f^{\prime}(0)} \int_{\mathbb{D} \backslash \mathcal{Z}(f)}|f(\zeta)|^{p-2} f(\zeta) \mathrm{d} \mu_{\alpha}(\zeta),
$$

where $\mathcal{Z}(f)$ is the zero set of $f$. The function $f$ is not identically zero by hypothesis, thus $\mu_{\alpha}(\mathcal{Z}(f))=0$ and

$$
\int_{\mathbb{D}}|f(\zeta)|^{p} \zeta \mathrm{d} \mu_{\alpha}(\zeta)=\frac{p}{2(\alpha+2)} \overline{f^{\prime}(0)} \int_{\mathbb{D}}|f(\zeta)|^{p-2} f(\zeta) \mathrm{d} \mu_{\alpha}(\zeta)
$$

Acknowledgements The author is partially supported by grant PID2019-106870GB-I00 from Ministerio de Ciencia e Innovación (Spain) and by Ministerio de Universidades Fellowship, reference number FPU17/00040.

Funding Open Access funding provided thanks to the CRUE-CSIC agreement with Springer Nature. The author is partially supported by grant PID2019-106870GB-I00 from MICINN (Spain) and by MU Fellowship, reference number FPU17/00040. 
Availability of data and material Not applicable.

Code availability Not applicable.

\section{Declarations}

Conflict of interest The author(s) declares that they have no conflicts of interest.

Open Access This article is licensed under a Creative Commons Attribution 4.0 International License, which permits use, sharing, adaptation, distribution and reproduction in any medium or format, as long as you give appropriate credit to the original author(s) and the source, provide a link to the Creative Commons licence, and indicate if changes were made. The images or other third party material in this article are included in the article's Creative Commons licence, unless indicated otherwise in a credit line to the material. If material is not included in the article's Creative Commons licence and your intended use is not permitted by statutory regulation or exceeds the permitted use, you will need to obtain permission directly from the copyright holder. To view a copy of this licence, visit http://creativecommons.org/licen ses/by/4.0/.

\section{References}

1. Anderson, J.M., Clunie, J., Pommerenke, C.H.: On Bloch functions and normal functions. J. Reine Angew. Math. 270, 12-37 (1974)

2. Arazy, J., Fisher, S.D., Peetre, J.: Möbius invariant function spaces. J. Reine Angew. Math. 363, 110-145 (1985). https://doi.org/10.1007/BFb0078341

3. Bayart, F., Brevig, O.F., Haimi, A., Ortega-Cerdà, J., Perfekt, K.-M.: Contractive inequalities for Bergman spaces and multiplicative Hankel forms. Trans. Am. Math. Soc. 371(1), 681-707 (2019). https://doi.org/10.1090/tran/7290

4. Duren, P.L.: Theory of $H^{p}$ Spaces, Pure and Applied Mathematics, vol. 38. Academic Press, New York-London (1970)

5. Duren, P.L., Schuster, A., Spaces, B.: Mathematical Surveys and Monographs. American Mathematical Society, Providence (2004). https://doi.org/10.1090/surv/100

6. Hedenmalm, H., Korenblum, B., Zhu, K.: Theory of Bergman Spaces, Graduate Texts in Mathematics. Springer, New York (2000). https://doi.org/10.1007/978-1-4612-0497-8

7. Pommerenke, Ch.: Boundary Behaviour of Conformal Maps, Grundlehren der Mathematischen Wissenschaften [Fundamental Principles of Mathematical Sciences]. Springer, Berlin (1992). https://doi.org/10.1007/978-3-662-02770-7

8. Rubel, L.A., Timoney, R.M.: An extremal property of the Bloch space. Proc. Am. Math. Soc. 75(1), 45-49 (1979). https://doi.org/10.2307/2042668

9. Vukotić, D.: A sharp estimate for $A_{\alpha}{ }^{p}$ functions in $C^{n}$. Proc. Am. Math. Soc. 117(3), 753-756 (1993). https://doi.org/10.2307/2159138

10. Zhu, K.: Operator Theory in Function Spaces. In: Second, Mathematical Surveys and Monographs. American Mathematical Society, Providence (2007). https://doi.org/10.1090/surv/138 